\title{
Large-eddy simulation study of turbulent flow around a rectangular spur dike
}

\author{
Seokkoo Kang ${ }^{1, *}$ \\ ${ }^{1}$ Department of Civil and Environmental Engineering, Hanyang University, Seoul, Republic of Korea
}

\begin{abstract}
High-resolution large-eddy simulation (LES) is carried out for investigating three-dimensional flow fields around a rectangular spur dike installed in an open-channel flume. The LES showed good agreement with the measurement obtained using Acoustic Doppler Velocimetry. Analysis of the LES result shows that the flow structure around and in the wake of the spur dike is highly complex and three-dimensional. Namely, flow upstream of the spur dike is featured by a vortex system near the bed, another vortex system beneath the free surface, and a recirculation region in front of the spur dike. All of these flow structures are laterally oriented. Moreover, flow in the wake region consists of a large vertically oriented recirculation region and a smaller laterally oriented recirculation region near the bottom corner downstream of the spur dike.
\end{abstract}

\section{Introduction}

One of the widely used approaches for restoring a stream from the point view of fluvial hydraulics and geomorphology has been the installation of instream structures near the streambank, in such a way that they alter the flow and sediment transport processes to improve water quality, to increase biodiversity, to enhance fish habitat, or to improve channel and bank stability. Several kinds of streambank-attached instream structures have been proposed until now, and they include the Iowa vane [1], a single-arm rock vane and its variants (cross vane, J-hook vane, W-weir, etc.) [2, 3], and a spur dike (also known as a bendway weir, groyne, or barb) $[4,5]$. The spur dike, among others, has received considerable attention from researchers because of its geometrical simplicity, which makes its installation easier in both field and laboratory. Many of the early studies on this structure (see for instance, [6-8]) have focused on the observation of scour and deposition patterns instream structures, but recent studies have been directed toward understanding the turbulent flow characteristics and three-dimensional flow fields using laboratory experiments or numerical simulations.

A number of experimental studies have been conducted for measuring mean velocities and turbulence statistics around a spur dike using Acoustic Doppler Velocimetry (ADV) [9, 10] or Particle Image Velocimetry (PIV) [11]. Kuhnle et al. [9] measured the three-dimensional flow velocities around a submerged, trapezoidal-shape spur dike in a laboratory flume using ADV. Duan [10] measured the three-dimensional velocities, turbulence kinetic energy, and Reynolds shear stresses around a non-submerged, rectangular spur dike using ADV. Higham et al. [11] measured two-dimensional velocity field and Reynolds stresses in the wake of

\footnotetext{
${ }^{*}$ Corresponding author; e-mail: kangsk78@hanyang.ac.kr
} 
a groyne using PIV. Although some experimental studies have presented measurements of velocity fields and turbulent quantities, they have also limitations in that: 1) the spatial resolution of the measurements were not high enough to reveal the three-dimensional structure of the flow, or only two-dimensional velocity fields were obtained; and 2) the measurements were done mostly in the vicinity of instream structures or scour hole. As a result, most of the previous experimental studies failed to present the detailed structure of three-dimensional velocity fields and associated turbulence statistics in both near and far wakes. This is, to some extent, due to the fact that a very long time is required for acquiring a sufficient number of velocity measurements for the reconstruction of detailed three-dimensional velocity fields. It is important to emphasize that the measurement takes much longer especially when a spur dike is not submerged, which is because the size of the wake behind a spur dike becomes substantially larger compared to the submerged case. Consequently, few experimental studies have reported detailed measurements of three-dimensional velocity and turbulent stresses in the entire wake of a non-submerged spur dike. To my best knowledge, this kind of experiment has been conducted only recently by Jeon et al. [12]. They investigated the flow around a spur dike installed in a laboratory flume by measuring the three-dimensional velocity field and free surface elevations in the whole of the recirculation region that is formed downstream of the spur dike.

Numerical simulations have been also employed for studying the flow around a nonsubmerged instream structure as they circumvent the difficulties of obtaining a large number of velocity measurements. Particularly, computational models based on advanced turbulence closure methods such as large-eddy simulation (LES) and detached-eddy simulation (DES), which are capable of resolving coherent structures in turbulence, have been applied to simulate the flow around an instream structure under non-submerged condition [13-15]. Most of the studies using DES or LES, however, have focused on studying instantaneous coherent flow structures of a horseshoe vortex system and shear layers in the vicinity of instream structures, rather than time-averaged flow structure and turbulence statistics in the wake regions.

This study is motivated by the need to improve understanding of the structure of threedimensional flow fields around and in the wake of a spur dike under non-submerged conditions and its associated turbulence mechanisms, which are largely unknown to date. To that end, LES is conducted for the recent experimental study performed by Jeon et al. [12], where several thousands of velocity measurements and flow visualizations were performed. Although various interesting flow phenomena have been newly discovered from the measured three-dimensional flow field in that study, the flow field in the vicinity of free surfaces and side walls could not be obtained due to the inability of the ADV to measure these regions. Furthermore, several thousands of measurement points are still not sufficient to examine the detailed flow structures throughout the whole wake. This study, as the continuation of the research performed by Jeon et al. [12], aims to further elucidate the structure of the threedimensional flow fields around and in the whole wake of the spur dike and their associated turbulence characteristics by investigating the same flow configuration using numerical simulation.

\section{Computational model}

Numerical simulation is carried out using the LES model developed by Kang et al. [16], which solves the spatially-filtered Navier-Stokes equations and continuity equations closed with the dynamic Smagorinsky model [17] formulated in generalized curvilinear coordinates. The details of the governing equations and numerical methods are omitted for the sake of brevity, as they can be found in [16]. 
An important feature of the present numerical model is the capability to handle complex geometries by using the Curvilinear Immersed Boundary (CURVIB) method [18]. In the CURVIB method, a moving/stationary immersed boundary (channel bathymetry and/or instream structures) is embedded in a background, computational domain (river or flume) that is discretized with a boundary-fitted curvilinear mesh. Rather than using a boundary conforming mesh to describe the immersed moving boundary, the CURVIB method treats the boundary as a sharp interface, and boundary conditions are reconstructed at curvilinear grid nodes in the immediate vicinity of the boundary using interpolation along the local normal to the boundary direction [16].

\section{Description of flow problem}

Jeon et al. [12] conducted two flume experiments with the same flow depth, but with different flow rates. In this study, the LES is carried out for the lower flow rate case, where free surface deformation was almost negligible. The flow and geometrical parameters are summarized in Table 1 . Of the listed parameters, $L / H$ determines the shallowness (or two-dimensionality) of the flow near non-submerged obstacles. Jeon et al. [12] showed that the measured velocity fields exhibited strong three-dimensional flow structures at the present value of $L / H=1.4$. Therefore, a similar trend is expected in the LES.

Table 1. Flow and geometrical parameters. $g$ denote the acceleration due to gravity. $u_{\tau}$ is obtained from the approach flow.

Flow and geometrical parameters

\begin{tabular}{lc}
\hline Flow depth at the tailgate $(H)$ & $0.215 \mathrm{~m}$ \\
Flume width $(B)$ & $0.9 \mathrm{~m}$ \\
Streamwise length of the spur dike $(t)$ & $0.04 \mathrm{~m}$ \\
Cross stream length of the spur dike $(L)$ & $0.3 \mathrm{~m}$ \\
Length-to-depth ratio $(L / H)$ & 1.4 \\
Volume flow rate $(Q)$ & $2.78 \times 10^{-2} \mathrm{~m}^{3} / \mathrm{s}$ \\
Mean velocity $\left(U_{0}\right)$ & $0.144 \mathrm{~m} / \mathrm{s}$ \\
Froude number $\left(F r=U_{0} / \sqrt{g H}\right)$ & 0.10 \\
Kinematic viscosity $(v)$ & $1.03 \times 10^{-6} \mathrm{~m}^{2} / \mathrm{s}$ \\
Reynolds number $\left(R e=U_{0} H / v\right)$ & $3.00 \times 10^{4}$ \\
Friction velocity $\left(u_{\tau}\right)$ & $0.007 \mathrm{~m} / \mathrm{s}$ \\
\hline
\end{tabular}

\section{Computational details}

The dimensionless coordinates and computational domain with the embedded immersed body, representing the spur dike, are shown in Figure 1. The $x, y$, and $z$ directions indicate the streamwise, spanwise, and vertical directions, respectively. The dimensions of the computational domain are $21.67 L(6.5 \mathrm{~m}), 3 L(0.9 \mathrm{~m})$, and $H(0.21 \mathrm{~m})$ in the $x, y$, and $z$ directions, respectively. The streamwise length of the domain was set sufficiently large to include the whole recirculation region formed downstream of the spur dike.

Table 2 shows the details of computational parameters. Approximately 57 million grid nodes were employed in the calculation. The minimum grid spacing of approximately 3 wall units were used near the bed $(z=0)$ and two side walls $(y=0$ and $0.9 \mathrm{~m})$ in their normal directions. The computational time step was chosen as $0.003 \mathrm{~s}$, which corresponds to the dimensionless time step of $\Delta t^{+}=u_{\tau}^{2} t / v=0.15$. 


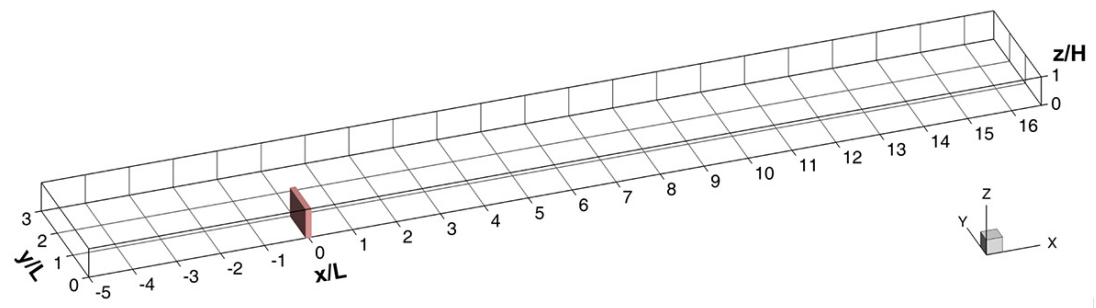

Figure 1. The coordinates and computational domain. The rectangular block centered at $x=0$ is the immersed body representing the spur dike.

As very little free surface deformation was observed in the experiment, the rigid-lid approximation, which treats the free surface as a shear-free plane surface, is employed at the top boundary. At the inlet $(x / L=-5)$, instantaneous three-dimensional velocity fields extracted from the separate LES, solving fully-developed turbulent flow with streamwise periodicity, is specified. At the outlet $(x / L=-16.67)$, zero-gradient of the velocity is imposed. At the bed $(z=0)$, side wall $(y / L=0$ and 3$)$ boundaries, and the immersed boundary nodes, a near-wall modeling is employed.

Table 2. Details of the computational parameters. The superscript ${ }^{+}$denotes the viscous length and time scales made dimensionless using $v$ and $u_{\tau} . l^{+}=u_{\tau} l / v(l$ is $x, y$, or $z)$ and $t^{+}=u_{\tau}^{2} t / v . \Delta$ denotes grid spacing.

Computational parameters

\begin{tabular}{lc}
\hline$N_{x} \times N_{y} \times N_{z}$ & $811 \times 483 \times 146$ \\
Total number of nodes & $5.71 \times 10^{7}$ \\
$\Delta x ; \Delta x^{+}$ & $0.0025-0.0380 \mathrm{~m} ; 18-266$ \\
$\Delta y, \Delta z ; \Delta y^{+}, \Delta z^{+}$ & $0.0004-0.0025 \mathrm{~m} ; 3-18$ \\
$\Delta t ; \Delta t^{+}=u_{\tau}^{2} \Delta t / v$ & $0.003 \mathrm{~s} ; 0.15$ \\
\hline
\end{tabular}

\section{LES results}

In this section, the results of the LES are firstly validated through comparisons with the experimental data [12], and the LES-computed flow fields are subsequently analyzed to explore the flow structure and turbulence characteristics around and in the wake of the spur dike. Some variables and notations used in this section are defined as follows. $u, v$, and $w$ are the instantaneous velocity components in the $x, y$, and $z$ direction, respectively. \langle\rangle means the mean (time-averaged) component, and the superscript ' indicates the temporally fluctuating component of any variable, i.e., $f=f^{\prime}+\langle f\rangle$ for any variable $f$. $k$ is the turbulence kinetic energy defined as $k=\frac{1}{2}\left(\left\langle u^{\prime} u^{\prime}\right\rangle+\left\langle v^{\prime} v^{\prime}\right\rangle+\left\langle w^{\prime} w^{\prime}\right\rangle\right)$. Other variables are listed in Table 1 .

In Figure 2, time-averaged turbulence statistics computed by the LES are compared with the experiment. The comparisons are made for the profiles of dimensionless $\langle u\rangle,\langle v\rangle, k$, and $\left\langle u^{\prime} v^{\prime}\right\rangle$ along the transverse lines in the mid-depth plane at $x / L=-3.3,-0.9,1.67,3.33$, and 13.33. It is seen that the LES accurately predicts both the streamwise and transverse velocities, and also the turbulent stresses in regions around and in the wake of the spur dike. Particularly, the presence and location of inflection points, indicating the formation of shear layers, in the $\langle u\rangle$ and $\langle v\rangle$ profiles are accurately predicted at $x / L=1.67$ and 3.33. Moreover, the profiles of $k$ and the $\left\langle u^{\prime} v^{\prime}\right\rangle$ Reynolds stress are also predicted well. In short, the LES predictions are satisfactory both quantitatively and qualitatively. 

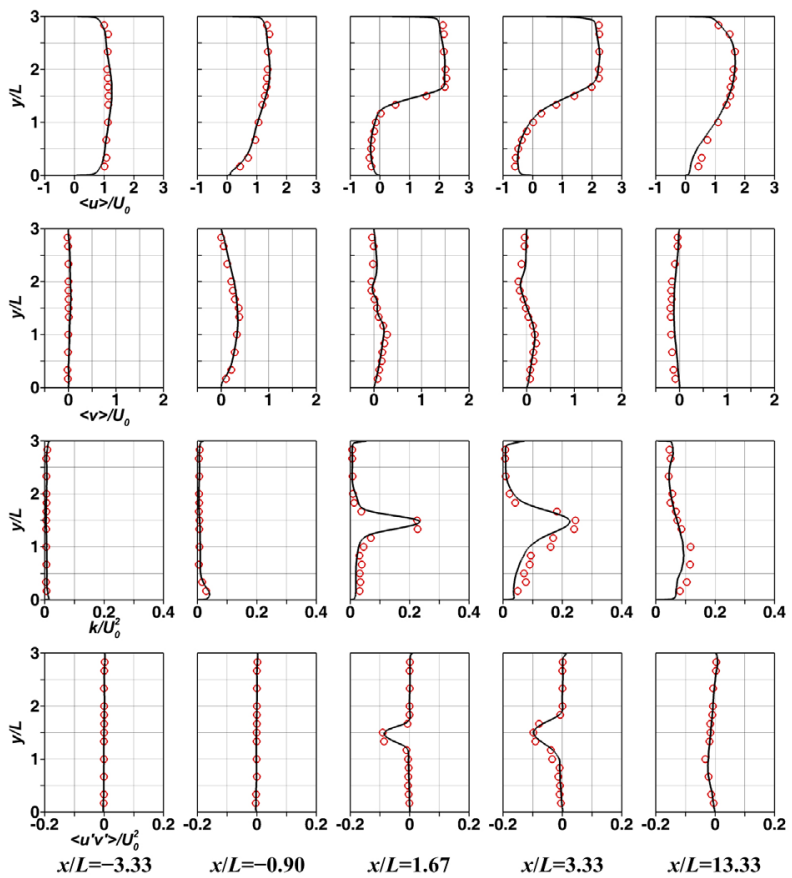

Figure 2. Comparisons of the computed (lines) and measured (symbols) transverse profiles of mean velocities turbulence quantities in the mid-depth plane at various $x$ locations.

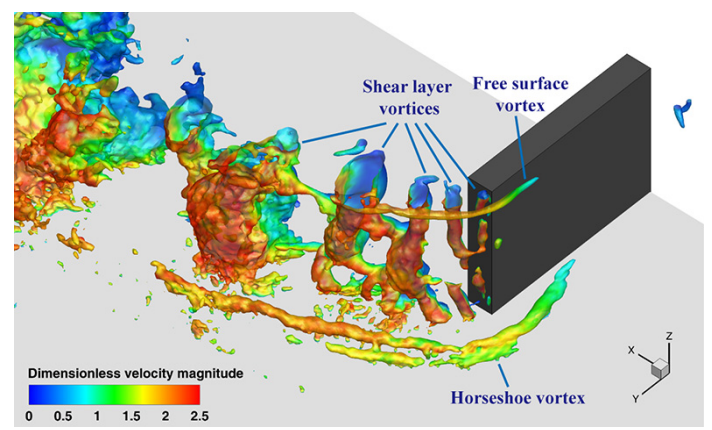

Figure 3. The isosurfaces of $p^{\prime} / U_{0}^{2}=-0.1$ showing instantaneous vortical structures around the spur dike. Contours indicate the instantaneous dimensionless velocity magnitude, $\sqrt{u^{2}+v^{2}+w^{2}} / U_{0} . x$ is the flow direction.

In what follows, three-dimensional structure of the computed, instantaneous and mean flow is explored.

Instantaneous flow patterns are investigated in Figure 3. This figure shows isosurfaces of a negative pressure fluctuation. Negative pressure fluctuations are associated with the locations of coherent vortices [19] and they reveal large-scale vortical structures. Three distinct kinds of flow structures are identified: 1) the laterally oriented horseshoe vortex system in the lower front of the spur dike; 2) the vertically oriented multiple shear layer vortices; 3 ) the laterally oriented vortex system under the free surface. Both the horseshoe vortex near the bed and similar-shaped vortex near the free surface are found to move in the downstream direction 
around the upstream face of the spur dike and form two different arch-shaped trajectories. In addition, the vertically oriented shear layer vortices are generated and shed from the edge between the upstream and side surfaces of the spur dike. These vortices remain coherent only for a short distance and grow in size and lose coherence while moving downstream. In regions directly downstream of the spur dike, no significant large-scale vortical structure is observed.
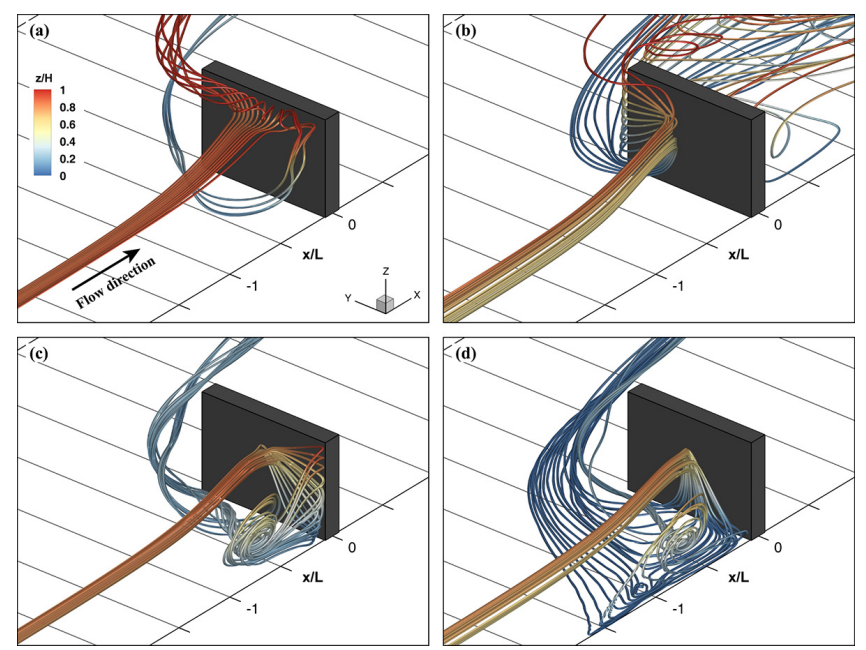

Figure 4. Streamlines of the time-averaged flow in front of the spur dike.

Three-dimensional structure of the mean flow is investigated in Figure 4, which shows streamlines of the time-averaged flow originated from regions upstream of the spur dike. The streamlines are colored by the dimensionless vertical coordinate $(z / H)$. The blue and red colors indicate regions near the bed and free surface, respectively. It is shown that the incoming streamlines collide on the upstream face of the spur dike and expand radially on its surface in all directions. Figure 4(a) shows that part of these streamlines move upward to form a spiral flow structure under the free surface, which is same as the laterally oriented free surface vortex that was observed in Figure 3. Near the free surface, the streamlines diverge horizontally toward and away from the side wall $(y=0)$. The streamlines moving away from the side wall ultimately form an arch-shaped trajectory downstream of the spur dike, while those moving toward the side wall form downward flow along the side wall and converge to another arch-shaped trajectory near the bed. Figure 4(b) shows that the streamlines moving horizontally toward the center of the flume after the collision finally separate from the surface to form a recirculation zone in the wake. As can be seen, streamline patterns in the wake is highly three-dimensional and complex. In both Figures 4(c) and 4(d), a laterally oriented recirculation zone is observed near the side wall and in front of the spur dike. The axis of rotation of this zone is perpendicular to the side wall, and the streamwise dimension of the zone is approximately $1.5 \mathrm{~L}$. The streamlines leaving the recirculating region form a spiral flow pattern around the spur dike and near the bed, which is the footprint of the horseshoe vortex.

Time-averaged streamlines in the wake of the spur dike are plotted in Figure 5. As can be seen from Figure 5(a), the structure of the three-dimensional flow field in the wake is extremely intricate. A vertically oriented primary recirculation zone, rotating clockwise around the $z$ axis, is observed to dominate the whole wake region. A smaller laterally oriented recirculation zone, which rotates counterclockwise around the $y$ axis, is observed near the corner 


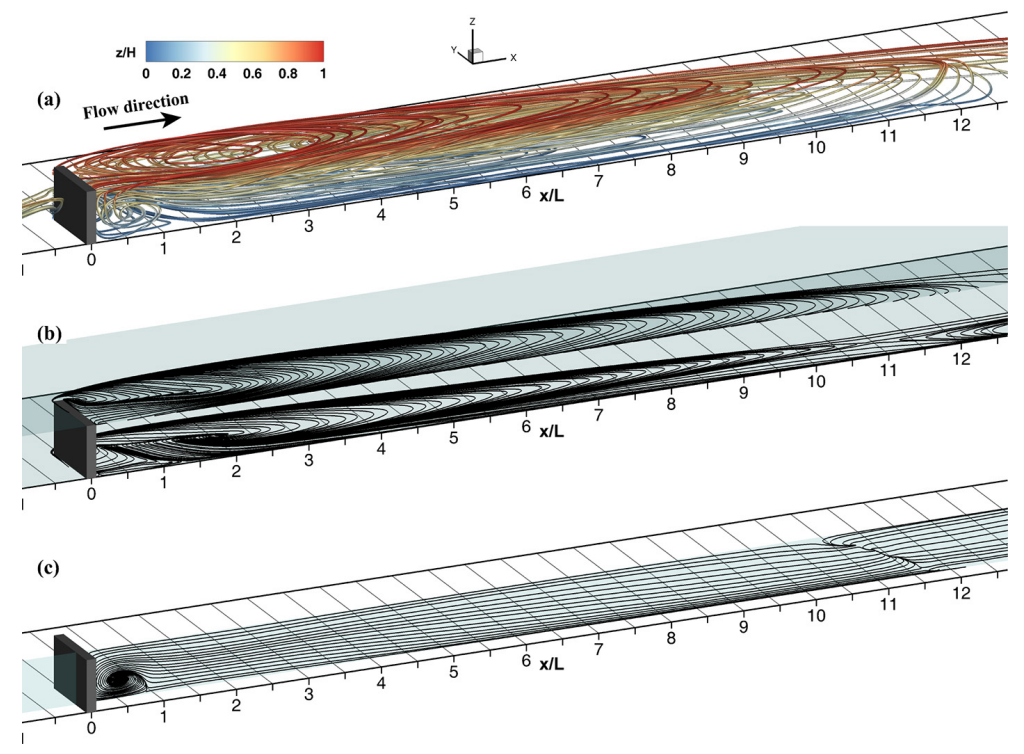

Figure 5. Streamlines of the time-averaged flow in the wake regions. (a) three-dimensional streamlines; (b) limiting streamlines on the surfaces of the planes near the bed $(z / H=0.01)$ and at the free surface $(z / H=1)$; (c) limiting streamlines on the surface of the plane $y / L=0.33$.

between the downstream face of the spur dike and bed. To better visualize the wake structure, limiting streamlines are plotted on the surfaces of $x y$ and $x z$ planes in Figure 5(b) and 5(c), respectively. The comparison between the streamlines at the planes of $z / H=0.01$ and 1 (see Figure 5(b)) clearly show that the centers of the recirculation eddies are located differently from each other. At the free surface, the center is located very close to the spur dike; while near the bed, it is located at approximately $x / L=2$. Moreover, the limiting streamline patterns changes significantly with different elevations, which suggests that flow in the wake is highly three-dimensional. The limiting streamlines at the $y / L=0.33$ plane (see Figure 5(c)) clearly reveals the presence of the corner recirculation zone. The shown streamline patterns indicate that the corner recirculation region is caused by the collision of the upstream directed flow, induced by the primary recirculation zone, on the downstream face of the spur dike.

The above results show that the mean flow structure around and in the wake of the spur dike are strongly three-dimensional. The present observations are contrary to those of Paik and Sotiropoulos [13] who studied flow past a side wall-mounted rectangular obstacle with much larger $L / H$ of 27 using DES. They found that except a narrow region in front of the obstacle, mean vertical velocities were nearly zero, i.e., velocity patterns were two-dimensional. A stronger three-dimensionality observed in the present case is due to the lower value of $L / H$, which determines the shallowness of flow around a non-submerged obstacle [20].

\section{Summary and conclusions}

In this paper, high-resolution LES was carried out for investigating three-dimensional structure of the flow around a non-submerged spur dike with a low length-to-depth ratio. Satisfactory agreement was observed between the computed and measured turbulence statistics.

The LES-computed flow field revealed the detailed three-dimensional structure of flow, and several important flow features were observed. In regions upstream of the spur dike, the 
incoming streamlines collide on the upstream face of the spur dike and expand radially on the surface in all directions. The streamlines are subsequently connected to: 1) the laterally oriented vortex system below the free surface; 2) the laterally oriented recirculation region near the sidewall; and 3) the outer boundary of the downstream wake after the separation from the upstream surface of the spur dike. Flow in the wake region consists of the two different recirculation regions with different orientations: 1) the vertically oriented primary recirculation zone which occupies almost the whole wake region; and 2) the laterally oriented, corner recirculation region near the corner between the downstream face of the spur dike and flume bed.

Owing to the limited length of this paper, three-dimensional flow structure around the spur dike and associated turbulence characteristics have not been fully explained. Further studies are underway to examine the turbulence characteristics and to elucidate the topology of the three-dimensional flow structure in greater detail.

\section{References}

[1] A.J. Odgaard, J.F. Kennedy, J. Hydraul. Eng. 109, 1161 (1983)

[2] D.L. Rosgen, Proceedings, Wetland Engineering and River Restoration Conference CDROM, ASCE, Reston, VA. (2001)

[3] P.A. Johnson, R.L. Tereska, E.R. Brown, J Am Water Resour Assoc. 38, 1143 (2002)

[4] D.L. Derrick, The bendway weir: An in-stream erosion control and habitat improvement structures for the 1990's, in Proceedings of the International Erosion Control Association's 27th Annual Conference and Trade Exposition (Seattle, WA, USA, 1996).

[5] R. Ettema, G. Kirkil, M. Muste, J. Hydraul. Eng. 132, 33 (2006)

[6] R.A. Kuhnle, C.V. Alonso, F.D. Shields, J. Hydraul. Eng. 125, 972 (1999)

[7] U. Kothyari, K. Ranga Raju, J. Hydraul. Res. 39, 367 (2001)

[8] R. Ettema, M. Muste, J. Hydraul. Eng. 130, 635 (2004)

[9] R.A. Kuhnle, Y. Jia, C.V. Alonso, J. Hydraul. Eng. 134, 916 (2008)

[10] J.G. Duan, J. Hydraul. Eng. 135, 803 (2009)

[11] J. Higham, W. Brevis, C. Keylock, A. Safarzadeh, Adv. Water Resour. (2017)

[12] J. Jeon, J. Lee, S. Kang, Water Resour. Res (in press)

[13] J. Paik, F. Sotiropoulos, Physics of Fluids 17, 115104 (2005)

[14] M. Koken, G. Constantinescu, Water Resour. Res 44 (2008)

[15] M. Koken, G. Constantinescu, Water Resour. Res 47 (2011)

[16] S. Kang, A. Lightbody, C. Hill, F. Sotiropoulos, Adv Water Resour 34, 98 (2011)

[17] M. Germano, U. Piomelli, P. Moin, W.H. Cabot, Phys. Fluids A 3, 1760 (1991)

[18] L. Ge, F. Sotiropoulos, J. Comput. Phys. 225, 1782 (2007)

[19] S. Kang, C. Hill, F. Sotiropoulos, Water Resour. Res 52, 7869 (2016)

[20] D. Chen, G.H. Jirka, Fluid Dyn. Res 16, 11 (1995) 